\title{
Clostridium butyricum protects intestinal barrier function via upregulation of tight junction proteins and activation of the Akt/mTOR signaling pathway in a mouse model of dextran sodium sulfate-induced colitis
}

\author{
MIAO LIU ${ }^{1-3 *}$, WENJIE XIE ${ }^{3,4^{*}}$, XINYUE WAN ${ }^{1-3}$ and TAO DENG ${ }^{1-3}$ \\ ${ }^{1}$ Department of Gastroenterology, Renmin Hospital of Wuhan University; \\ ${ }^{2}$ Key Laboratory of Hubei Province for Digestive System Disease; ${ }^{3}$ Central Laboratory of Renmin Hospital; \\ ${ }^{4}$ Department of Critical Care Medicine, Renmin Hospital of Wuhan University, Wuhan, Hubei 430060, P.R. China
}

Received February 6, 2020; Accepted July 1, 2020

DOI: $10.3892 /$ etm.2020.9138

\begin{abstract}
Clostridium butyricum ( $C B)$, a probiotic, is a gram-positive obligate anaerobic bacillus with acid and heat resistant properties. Previous studies have reported that $C B$ has beneficial effects in intestinal diseases and regulates intestinal function. The aim of the present study was to investigate the protective effects and mechanisms of $C B$ on the intestinal barrier function. Mice were randomly divided into three experimental groups ( $\mathrm{n}=15 \mathrm{mice} / \mathrm{group}$ ), including control, dextran sodium sulfate (DSS) and DSS $+C B$. In the DSS and DSS $+C B$ groups colitis was induced with $3 \%$ DSS dissolved in drinking water for 7 days. DSS $+C B$ group mice were co-treated daily with $200 \mu \mathrm{l}\left(2 \times 10^{8} \mathrm{CFU}\right) C B$ solution via gavage. The intestinal mucosal barrier function in mice was assessed by measuring FITC-labeled 4-kDa dextran (molecular weight, 4,000 Da) flux and by analyzing the expression of tight junction (TJ)-related proteins using western blot analysis. In addition, the secretion levels of tumor necrosis factor- $\alpha$ (TNF- $\alpha$ ), interleukin (IL)-1 $\beta$, IL-6, IL-10 and IL-13, and the concentration of malondialdehyde, glutathione and superoxide dismutase were detected using ELISAs to determine inflammation and oxidative stress, respectively. The activation status of the Akt/mTOR signaling pathway was also investigated using western blot analysis. The results demonstrated that, in mice with DSS-induced colitis treatment, co-treatment with $C B$ attenuated colitis symptoms and intestinal permeability,
\end{abstract}

Correspondence to: Professor Tao Deng, Department of Gastroenterology, Renmin Hospital of Wuhan University, 238 Jiefang Road, Wuchang, Wuhan, Hubei 430060, P.R. China E-mail: dt641120@163.com

${ }^{*}$ Contributed equally

Key words: Clostridium butyricum, intestinal barrier function, tight junctions, Akt/mTOR signaling pathway increased the expression levels of TJ-related proteins, decreased TNF- $\alpha$, IL-1 $\beta$ and IL-13 secretion levels but increased those of IL-10, and reduced oxidative stress. Additionally, $C B$ elevated the phosphorylation of Akt, mTOR and p70 ribosomal protein S6 kinase. Collectively, the present results indicated that $C B$ protected intestinal barrier function and decreased intestinal mucosal permeability via upregulating the expression levels of TJ-related proteins in a mouse model of DSS-induced colitis. Moreover, the results suggested that the effects of $C B$ could be mediated by the Akt/mTOR signaling pathway.

\section{Introduction}

The main function of the intestinal epithelium is to absorb water and nutrients to maintain life, and the transport of these substances should be tightly regulated in order to maintain homeostasis (1). The intestinal epithelium is an important structure, responsible for transporting water and nutrients, and constitutes a physical barrier regulating the selective paracellular permeability to ions and molecules (2). Adjacent intestinal epithelial cells are tightly packed together and are connected via three types of junctional complexes, namely desmosomes, adherens junctions and tight junctions (TJs) (3). TJs are the most apical junctions along the lateral surface, and are the main structure regulating epithelial barrier function (4). TJs are composed of transmembrane proteins, which include claudins, occludin and tricellulin, and cytosolic scaffold proteins, such as zona occludens proteins (ZO)1-3, Afadin (AF-6) and cingulin (2).

Ulcerative colitis (UC) is a chronic gastrointestinal disorder characterized by diffuse mucosal inflammation of the colon. In UC, pathological lesions are mainly located in the rectum, affecting a part or the whole large intestine (5). Moreover, the clinical course of the disease is characterized by remissions and exacerbations (6). It has been reported that dysregulation of the intestinal mucosal barrier contributes to the development, perpetuation and severity of inflammatory bowel disease (IBD) (7). At the same time, IBD may also contribute to a leaky epithelial barrier due to the inflammatory 
microenvironment (8). In UC, impaired complexity of TJs and downregulation of TJ-related proteins have been considered as the main mechanisms mediating the dysfunction of the intestinal mucosal barrier $(9,10)$. Commonly used drugs for UC treatment include 5-aminosalicylic acid, immunosuppressants and biological agents (5). Furthermore, given the continuous progress in the research on intestinal microorganisms, probiotics have become a novel treatment strategy for UC.

The gastrointestinal tract contains a large and complex ecology of microorganisms. For example, it is estimated that 100 trillion microbes reside in the human adult gut, collectively known as the gut microbiota (11). The mutually beneficial relationship between the host and its gut microbiota contributes to host health, as gut microbiota are involved in several biological processes, including the absorption of nutrients, protection against infections, maturation of the immune system and regulation of metabolism (12). The International Symposium of the International Scientific Association of Probiotics and Prebiotics defined probiotics as 'the living microorganisms that when administered in adequate amounts, confer health benefits on the host' (13). Clostridium butyricum $(C B)$ is a gram-positive obligate anaerobic bacillus. Compared with other non-sporulating probiotics, $C B$ is resistant to the effects of gastric acid and temperature, thus making its investigation and use easier $(14,15)$. It has been reported that butyrate, an important type of short chain fatty acid (SCFA) produced by $C B$, exhibits positive effects on the maintenance of intestinal barrier function $(16,17)$. Therefore, $C B$ is effectively used in clinical practice to improve the intestinal barrier function and to treat UC. However, its exact underlying mechanisms require further investigation.

In the present study, a dextran sodium sulfate(DSS)-induced colitis model was established in C57BL/6 mice to investigate the potential effects of $C B$ on the expression levels of TJ-related proteins, intestinal inflammation and oxidative stress. It has been well established that activation of the Akt/mTOR pathway and its downstream molecules, including $\mathrm{p} 70$ ribosomal protein S6 kinase (S6K) and eukaryotic translation initiation factor 4E binding protein 1 (4E-BP1), regulate protein synthesis (18). Therefore, the present study further investigated the role of $C B$ on the regulation of TJ-related protein expression levels via the Akt/mTOR pathway.

\section{Materials and methods}

Animals and mouse model. A total of 45 C57BL/6 mice (males; age, 6 weeks; weight, 16-20 g) were obtained from the Beijing Vital River Laboratory Animal Technology Co., Ltd. Animals were housed in a specific pathogen-free room at a temperature of $25^{\circ} \mathrm{C}$, a humidity of $45-50 \%$ and a $12 \mathrm{~h}$ light/dark cycle. All animals had free access to food and water, and were allowed to acclimatize to laboratory conditions for 7 days. All animal experiments were performed in accordance with the Guide for the Care and Use of Laboratory Animals and were approved by the Ethics Committee of Renmin Hospital of the Wuhan University.

Colitis was induced in C57BL/6 mice following treatment with 3\% DSS [molecular weight (MW), 35,000-50,000 Da; MP Biomedicals, LLC] dissolved in drinking water for 7 days, as previously described (19). The progression of DSS-induced colitis was monitored daily by measuring the disease activity index (DAI), as previously described (19). A freeze-dried powder containing $C B$ bacteria of $5 \times 10^{9}$ colony forming units $(\mathrm{CFU}) / \mathrm{g}$ was purchased from Kexing Biopharm Co., Ltd.

Experimental design. Mice were randomly divided into three experimental groups ( $\mathrm{n}=15 \mathrm{mice} / \mathrm{group}$ : Control, DSS and $\mathrm{DSS}+C B$ groups. In the DSS and DSS $+C B$ mice groups, animals were treated with $3 \%$ DSS dissolved in drinking water for 7 days, while mice in the control group received standard laboratory water ad libitum. During the induction of colitis, mice in the DSS $+C B$ group were co-treated daily with $200 \mu \mathrm{l}\left(2 \times 10^{8} \mathrm{CFU}\right) C B$ solution via gavage, as previously described (20). Mice in the control and DSS groups received $200 \mu \mathrm{l}$ normal saline via gavage. All mice were anesthetized with pentobarbital solution $(50 \mathrm{mg} / \mathrm{kg})$ via intraperitoneal injection 7 days after standard laboratory water or DSS treatment. Following anesthesia, $0.5 \mathrm{ml}$ blood was collected from the mice via eyeball enucleation. To isolate colon tissue, mice were sacrificed by cervical dislocation. Following a $2 \mathrm{~min}$ observation, mice were confirmed to be dead without respiration and corneal reflex. A $0.5-\mathrm{cm}$ segment of the distal colon was cut and isolated, rinsed with PBS, fixed for $48 \mathrm{~h}$ in $4 \%$ $(\mathrm{w} / \mathrm{v})$ paraformaldehyde at $4^{\circ} \mathrm{C}$, dehydrated and embedded in paraffin.

Intestinal permeability assay. The intestinal permeability of mice with DSS-induced colitis was measured as previously described (21). A total of $44 \mathrm{mg} / 100 \mathrm{~g}$ body weight FITC-labeled 4-kDa dextran (FD-4; MW, 4,000 Da; Sigma-Aldrich; Merck $\mathrm{KGaA}$ ) was administrated to each mouse via gavage following fasting for $4 \mathrm{~h}$. Following FD-4 administration for $5 \mathrm{~h}, 400 \mu \mathrm{l}$ blood was collected and centrifuged at 2,500 x g for $10 \mathrm{~min}$ at $4^{\circ} \mathrm{C}$ to isolate serum. The serum fluorescence intensity was determined using a multimode reader (PerkinElmer, Inc.) at excitation wavelength of $485 \mathrm{~nm}$ and emission wavelength of $520 \mathrm{~nm}$. Subsequently, a standard curve was generated using serial dilutions of FD-4.

Histology. Acolon segment was cut and isolated, its length was measured and analyzed using histology methods. Rectum tissues were fixed for $48 \mathrm{~h}$ with $4 \%$ (w/v) paraformaldehyde at $4^{\circ} \mathrm{C}$, dehydrated and embedded in paraffin. Subsequently, the tissue samples were sectioned (thickness, $4 \mu \mathrm{m}$ ), stained with hematoxylin and eosin (HE) for $97 \mathrm{~min}$ at room temperature and observed under an light microscope, at X100 and X200 magnification. Intestinal inflammation grading was performed as previously described (22). Histologic score was assessed in a blinded manner by two independent researchers.

Western blotting. To investigate the protein expression levels of claudin-1, claudin-2, occludin, and ZO-1, and the activation status of the Akt/mTOR signaling pathway, total protein samples were extracted from murine colon tissues using radioimmunoprecipitation assay lysis buffer (cat. no. P0013B; Beyotime Institute of Biotechnology), containing phenylmethanesulfonyl fluoride (cat. no. P105539; Shanghai Aladdin Bio-Chem Technology Co., Ltd.) and phosphoesterase inhibitors (cat. no. S1873; Beyotime Institute of Biotechnology). The concentration of the extracted proteins was measured using 
Table I. List of PCR primers and amplicon size.

\begin{tabular}{|c|c|c|c|}
\hline Gene name & Primer & Sequence & Size, bp \\
\hline \multirow[t]{2}{*}{ Mus GAPDH } & Forward & 5'-ATGGGTGTGAACCACGAGA-3' & 229 \\
\hline & Reverse & 5'-CAGGGATGATGTTCTGGGCA-3' & \\
\hline \multirow[t]{2}{*}{ Mus claudin-1 } & Forward & 5'-GGCTGATCGCAATCTTTGT-3' & 131 \\
\hline & Reverse & 5'-TAATGTCGCCAGACCTGAA-3' & \\
\hline \multirow[t]{2}{*}{ Mus claudin-2 } & Forward & 5'-CCGTGTTCTGCCAGGATT-3' & 199 \\
\hline & Reverse & 5'-GCTGAGATGATGCCCAAG-3' & \\
\hline \multirow[t]{2}{*}{ Mus occludin } & Forward & 5'-TGGATCTATGTACGGCTCAC-3' & 203 \\
\hline & Reverse & 5'-CCATCTTTCTTCGGGTTT-3' & \\
\hline \multirow[t]{2}{*}{ Mus ZO-1 } & Forward & 5'-CCAGCAACTTTCAGACCACC-3' & 154 \\
\hline & Reverse & 5'-TTGTGTACGGCTTTGGTGTG-3' & \\
\hline
\end{tabular}

ZO-1, zona occludens protein 1.

a bicinchoninic acid protein assay kit (Beyotime Institute of Biotechnology). Subsequently, total protein extracts were dissolved in 5X SDS-PAGE loading buffer and heated in a boiling water bath for $10 \mathrm{~min}$. A total of $40 \mu \mathrm{g}$ proteins/lane were loaded onto an SDS-PAGE (10\% gel) and electrophoresis was performed for $1.5 \mathrm{~h}$ prior to protein transfer onto PVDF membranes. Following transfer, membranes were blocked with $5 \%$ skimmed milk in TBS-0.1\% Tween 20 (TBST) buffer for $2 \mathrm{~h}$ at room temperature and then incubated with primary antibodies overnight at $4^{\circ} \mathrm{C}$. The following primary antibodies were used: Rabbit polyclonal anti-claudin-1 (cat. no. AF0127; 1:1,500), anti-claudin-2 (cat. no. AF0128; 1:1,500), anti-occludin (cat. no. DF7504; 1:1,500), anti-ZO-1 (cat. no. AF5145; 1:1,000; all from Affinity Biosciences, Inc.), anti-total-Akt (cat. no. 10176-2-AP; 1:1,000; ProteinTech Group, Inc.), anti-phosphorylated (p)-Akt (cat. no. 4060p; 1:1,000), anti-total-mTOR (cat. no. 2983; 1:1,000), anti-total-S6K (cat. no. 2708; 1:1,000), anti-p-S6K (cat. no. 9234; 1:1,000; all from Cell Signaling Technology, Inc.), anti-GAPDH (cat. no. AB-P-R001; 1:1,000; Hangzhou Goodhere Biotechnology Co., Ltd.). After washing five times with TBST for $5 \mathrm{~min}$ each, membranes were further immunoblotted with an HRP-conjugated AffiniPure goat anti-rabbit IgG (cat. no. BA1054; 1:50,000; Wuhan Boster Biological Technology, Ltd.) secondary antibody for $2 \mathrm{~h}$ at $37^{\circ} \mathrm{C}$. Subsequently, membranes were washed again with TBS-T for five times ( $5 \mathrm{~min} / \mathrm{time}$ ), treated with an ECL plus kit (cat. no. P1050; Applygen Technologies Inc.) for $\sim 5$ min at room temperature and the grayscale values of each band on the blots were analyzed with the BandScan v5.0 software (Glyko Biomedical Ltd.).

Reverse transcription-quantitative PCR (RT-qPCR). The mRNA expression levels of claudin-1, claudin-2, occludin and ZO-1 were determined using RT-qPCR. Colon tissues were homogenized and total RNA was extracted with TRIzol ${ }^{\circledR}$ reagent (cat. no. 15596-026; Ambion; Thermo Fisher Scientific, Inc.). Subsequently, total RNA was reverse transcribed into cDNA using a Hiscript RT reagent kit (cat. no. R101-01/02; Vazyme Biotech Co., Ltd.) under the following conditions: $25^{\circ} \mathrm{C}$ for $5 \mathrm{~min}, 50^{\circ} \mathrm{C}$ for $15 \mathrm{~min}, 85^{\circ} \mathrm{C}$ for $5 \mathrm{~min}$ and $4^{\circ} \mathrm{C}$ for $10 \mathrm{~min}$. The qPCR step was performed using a SYBR Green Master Mix kit (cat. no. Q111-02; Vazyme Biotech Co., Ltd.), according to the manufacturer's instructions, on a QuantStudio 6 Flex Real-Time PCR system (Applied Biosystems; Thermo Fisher Scientific, Inc.) under the following conditions: $50^{\circ} \mathrm{C}$ for $2 \mathrm{~min}, 95^{\circ} \mathrm{C}$ for $10 \mathrm{~min} ; 40$ cycles of $95^{\circ} \mathrm{C}$ for $30 \mathrm{sec}$ and $60^{\circ} \mathrm{C}$ for $30 \mathrm{sec}$. The data was analyzed with the $2^{-\Delta \Delta \mathrm{Cq}}$ method (23). GAPDH served as the endogenous control. The primer sequences and corresponding amplicon sizes are listed in Table I.

ELISA. Murine blood $(0.5 \mathrm{ml})$ was collected from the orbital venous plexus, as aforementioned, and centrifuged at $2,500 \mathrm{x} \mathrm{g}$ for $10 \mathrm{~min}$ at $4^{\circ} \mathrm{C}$. The supernatants were subsequently collected and stored at $-20^{\circ} \mathrm{C}$. The cytokines, TNF- $\alpha$ (cat. no. E-EL-M0049c), IL-1 $\beta$ (cat. no. E-EL-M 0037c), IL-6 (cat. no. E-EL-M0044c), IL-10 (cat. no. E-EL-M0037c) and IL-13 (cat. no. E-EL-M0727c) (all Elabscience, Inc.), were quantitatively measured using ELISA (Multiskan MK3; Thermo Fisher Scientific, Inc.) according to the manufacturer's protocols. The absorbance (optical density value) was measured on a microplate reader and the corresponding concentration of each sample was converted from the standard curve.

Statistical analysis. The experiments were repeated three times. Statistical analyses were performed using SPSS 20.0 software (IBM Corp.). Data are presented as the mean \pm SD. All data were analyzed using mixed and one-way ANOVA and followed by Tukey's post-hoc test. $\mathrm{P}<0.05$ was considered to indicate a statistically significant difference.

\section{Results}

$C B$ ameliorates DSS-induced colitis. To investigate the therapeutic efficacy of $C B$ treatment in DSS-induced colitis, the alterations in body weight of mice were detected. No significant changes in body weight were observed in the control group during the experiment (Fig. 1). However, mice in the DSS group exhibited significant weight loss from the 5th day, 


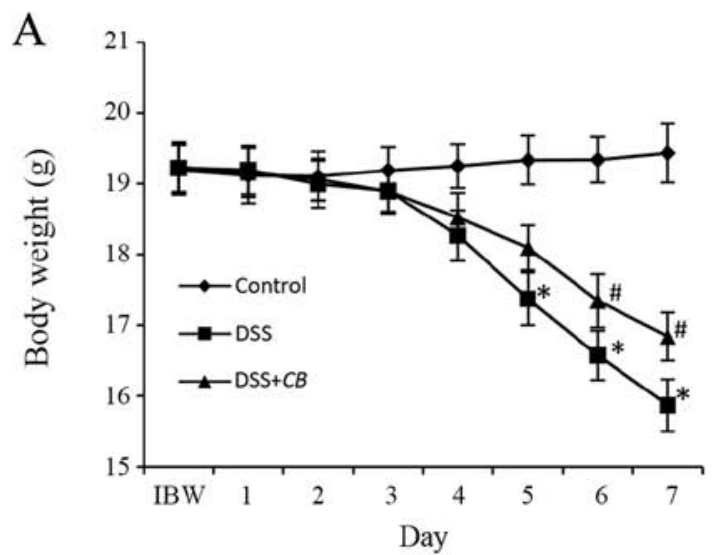

$\mathrm{C}$

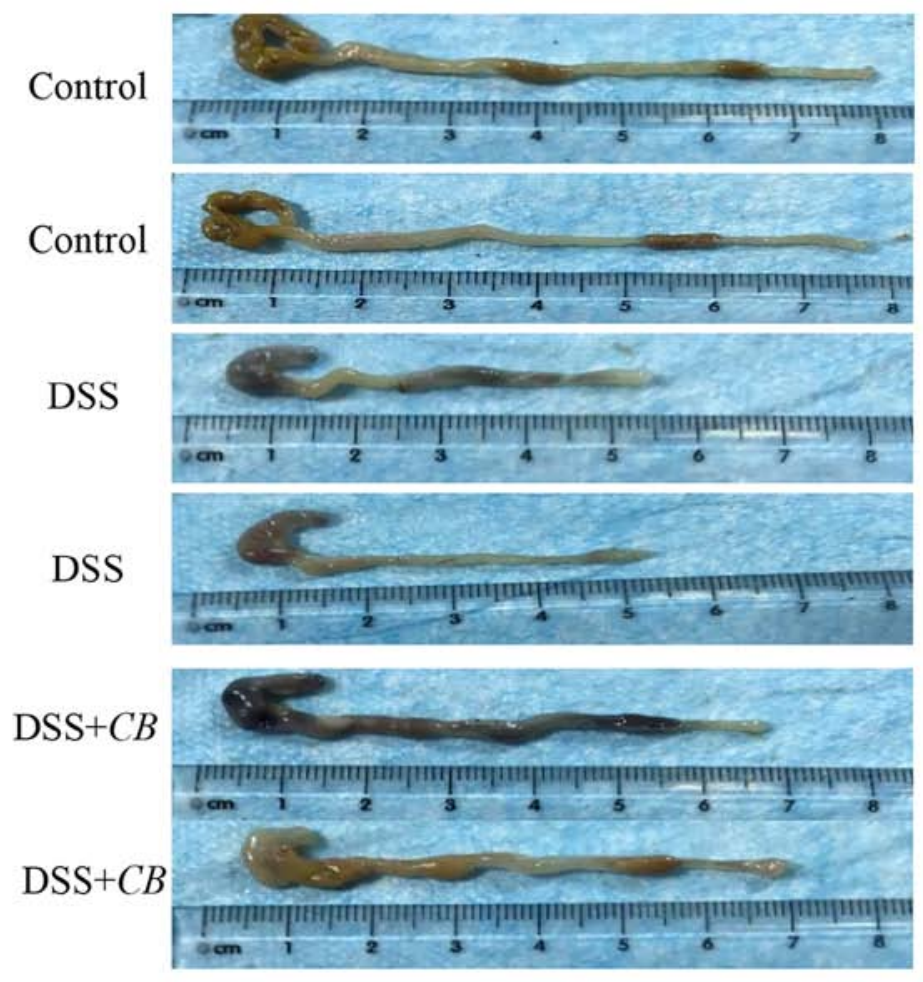

B

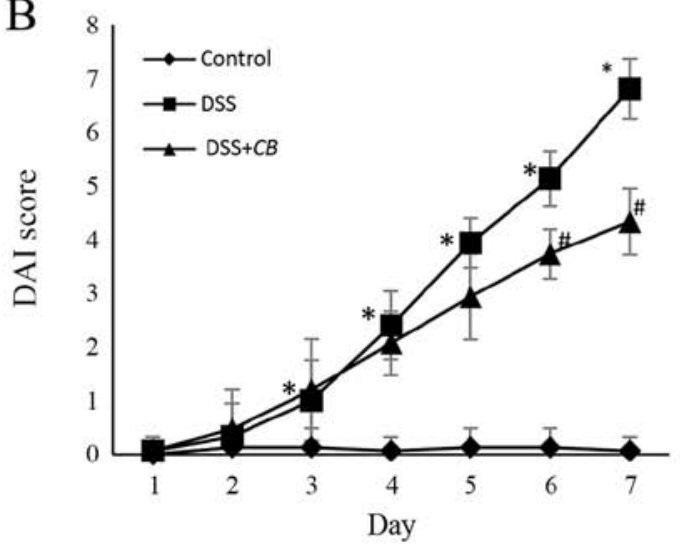

$\mathrm{D}$

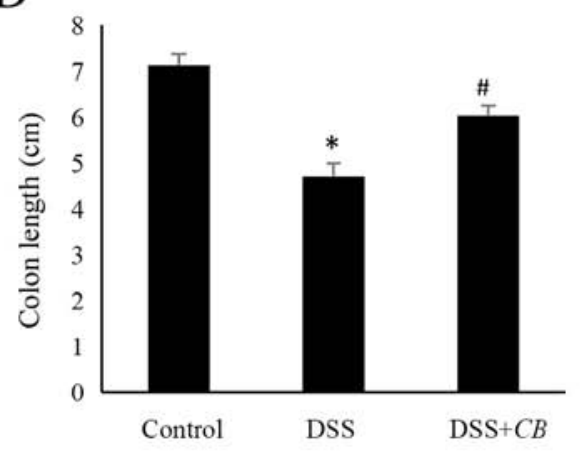

$\mathrm{E}$

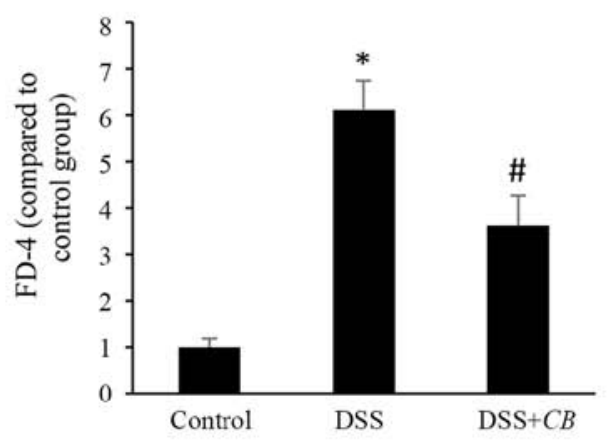

Figure 1. $C B$ treatment alleviates intestinal inflammation in DSS-induced colitis. Effect of $C B$ on (A) body weight and (B) DAI score, (C) colon length, which is quantified in (D), and (E) FD-4 permeability. Data are presented as the mean $\pm \mathrm{SD}$. ${ }^{*} \mathrm{P}<0.05$ vs. control group; ${ }^{*} \mathrm{P}<0.05$ vs. DSS group. IBW, initial body weight; $C B$, Clostridium butyricum; DSS, dextran sodium sulfate; DAI, disease activity index; FD-4, FITC-labeled 4-kDa dextran.

which was reversed following treatment with $C B$ from the 6th day. Subsequently, to assess the symptoms of DSS-induced colitis, the DAI score was determined in each group (Fig. 1B). The DAI score in the healthy control group was $\sim 0$ during the 7-day experimental period. However, mice in the DSS and DSS $+C B$ groups developed obvious symptoms of colitis, including activity reduction, diarrhea and rectal bleeding. These symptoms appeared at the 3rd day after colitis induction, reaching peak on the 7th day. Compared with the DSS group, mice in the DSS $+C B$ group also exhibited a significantly lower DAI score from day 6 . Furthermore, the colon length was significantly decreased in the DSS group compared with the control group; however, the colon length was significantly increased in the DSS $+C B$ group compared with the DSS group (Fig. $1 C$ and D). These findings indicated that $C B$ administration effectively alleviated DSS-induced colitis.
$C B$ restores intestinal barrier injury in the DSS-induced colitis mouse model. FD-4 was used to assess the intestinal permeability and the effects of $C B$ on intestinal barrier function. Mice in the DSS group demonstrated 6-fold increased FD-4 serum levels compared with those in the control group. This finding suggested that the intestinal barrier function was severely impaired in mice with DSS-induced colitis. Additionally, compared with the DSS group, serum FD-4 levels were 1.7-fold lower in the DSS $+C B$ group (Fig. 1E). The aforementioned data indicated that $C B$ improved the intestinal epithelial barrier function in DSS-induced colitis.

Effects of CB on colon histology in the DSS-induced colitis mouse model. HE staining demonstrated that $C B$ ameliorated the histopathologic changes in DSS-induced colitis. In the control group, the colonic mucosa was intact, glandular cells 
A

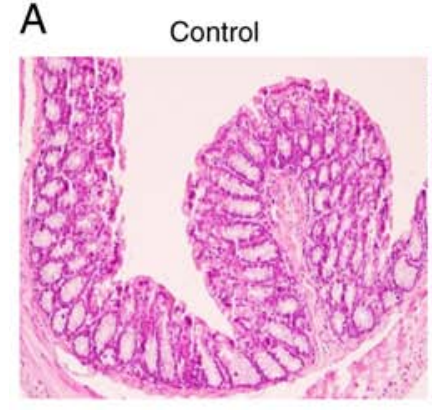

DSS

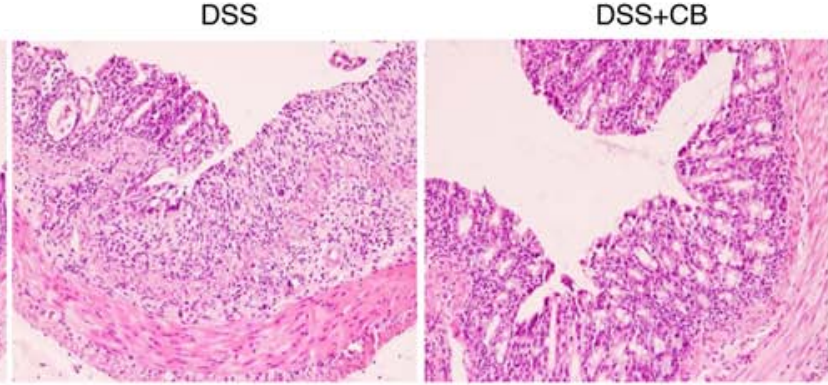

B

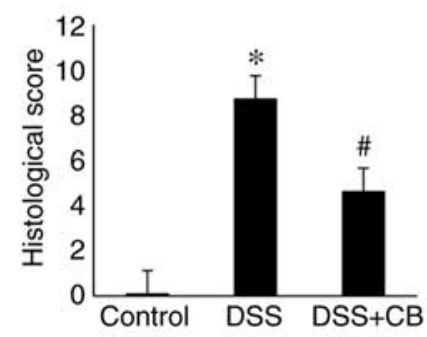

Figure 2. Effects of $C B$ on colon histology in the DSS-induced colitis mouse model. (A) Representative images of colon tissues from control, DSS and DSS $+C B$ groups (original magnification, $\mathrm{x} 200$ ). (B) Histological scores of colon tissue. Data are presented as the mean \pm SD. ${ }^{*} \mathrm{P}<0.05$ vs. control group; ${ }^{\prime} \mathrm{P}<0.05 \mathrm{vs}$. $\mathrm{DSS}$ group. DSS, dextran sodium sulfate; $C B$, Clostridium butyricum.

were arranged orderly and no inflammatory cell infiltration in the normal crypts was observed. However, compared with the control group, HE staining in the DSS group revealed epithelial defects, destroyed crypts and extensive inflammatory cell infiltration. Furthermore, compared with the DSS group, in the DSS $+C B$ group, the colonic mucosa injury was mild, with more intact glands and reduced number of inflammatory infiltrate cells apparent in the crypts (Fig. 2A). The histological score among the three groups was significantly different; the score was significantly increased in the DSS group compared with the control group; however, the score was significantly decreased in the DSS $+C B$ group compared with the DSS group (Fig. 2B).

Effects of $C B$ on intestinal inflammation and oxidative stress. The ELISA results indicated that treatment with DSS significantly elevated the secretion of proinflammatory cytokines, including TNF- $\alpha$, IL-1 $\beta$ and IL-13. However, the increased cytokine serum levels were reversed following co-treatment with $C B$. On the contrary, IL-10 secretion levels were attenuated in the DSS group compared with those in the control group, but its levels were increased in $C B$-treated mice. Moreover, compared with the control group, IL-6 secretion levels in the DSS group were significantly elevated, but there was no significant difference between the DSS and DSS $+C B$ group (Fig. 3A).

The results demonstrated that $C B$ treatment attenuated the DSS-induced alterations in lipid peroxidation and antioxidant enzymatic status in colon tissues. Therefore, following treatment with DSS, MDA levels were significantly increased, while those of GSH and SOD were decreased compared with the control group. However, co-treatment with $C B$ significantly restored the levels of MDA and GSH, and the enzymatic activity of SOD (Fig. 3B-D).

Effects of $C B$ on the expression levels of claudin-1, claudin-2, occludin and ZO-1. The effects of $C B$ on the protein and mRNA expression levels of the TJ-related proteins, claudin-1, claudin-2, occludin and ZO-1, were assessed using western blot analysis (Fig. 4A) and RT-qPCR (Fig. 4B), respectively. The protein expression levels of claudin-1, occludin and ZO-1 were decreased, and those of claudin- 2 were elevated in the DSS group compared with the control group. However, the expression levels of claudin-1, occludin, ZO-1 and claudin-2 were significantly reversed following co-treatment with $C B$. Consistent with the western blotting results, the relative mRNA synthesis findings obtained via RT-qPCR, indicated that $C B$ increased the mRNA expression levels of claudin-1, occludin and ZO-1, and decreased those of claudin-2 $(\mathrm{P}<0.05)$.

Effects of $C B$ on the activation of the Akt/mTOR signaling pathway. Further experiments were performed to investigate whether $C B$ promoted the expression levels of TJ-related proteins via the Akt/mTOR signaling pathway. The results demonstrated that in the DSS-induced colitis group p-Akt protein levels were significantly reduced compared with the control group; however, in the DSS $+C B$ group, p-Akt protein levels were significantly increased compared with the DSS group (Fig. 5). Subsequently, the protein expression levels of p-mTOR and p-S6K, two downstream molecules of the Akt/mTOR signaling pathway, were detected. Mice in the DSS group exhibited significantly decreased p-mTOR and p-S6K protein expression levels compared with the control group. However, pretreatment with $C B$ elevated both p-mTOR and $\mathrm{p}-\mathrm{S} 6 \mathrm{~K}$ protein expression levels, and the $\mathrm{p}-\mathrm{mTOR} / \mathrm{mTOR}$ and p-S6K/S6K ratios compared with the DSS group (Fig. 5). These findings suggested that DSS inhibited the activation of the Akt downstream signaling molecules, p-mTOR and S6K, while $C B$ restored the DSS-induced suppression of protein synthesis.

\section{Discussion}

UC and Crohn's disease are the two main forms of IBD which has been shown to have a complex genetic basis and is affected by several risk factors, including environmental factors, the intestinal microbiota and the host immune system (24). Moreover, increasing evidence has revealed that the impaired intestinal barrier is an important feature of colitis $(25,26)$. The gut microbiota and the host immune system constantly communicate with each other to maintain gut homeostasis and host health (24). $C B$ is a strictly anaerobic gram-positive bacterium, which produces acetic and butyric acid, two important components of SCFAs synthetized by gut microbiota (27). Furthermore, $C B$ commonly exists in the intestines of humans and animals, and has been proved effective to treat intestinal inflammation $(20,28,29)$. $C B$ also serves an important role in providing nutrients for the host and maintaining the 


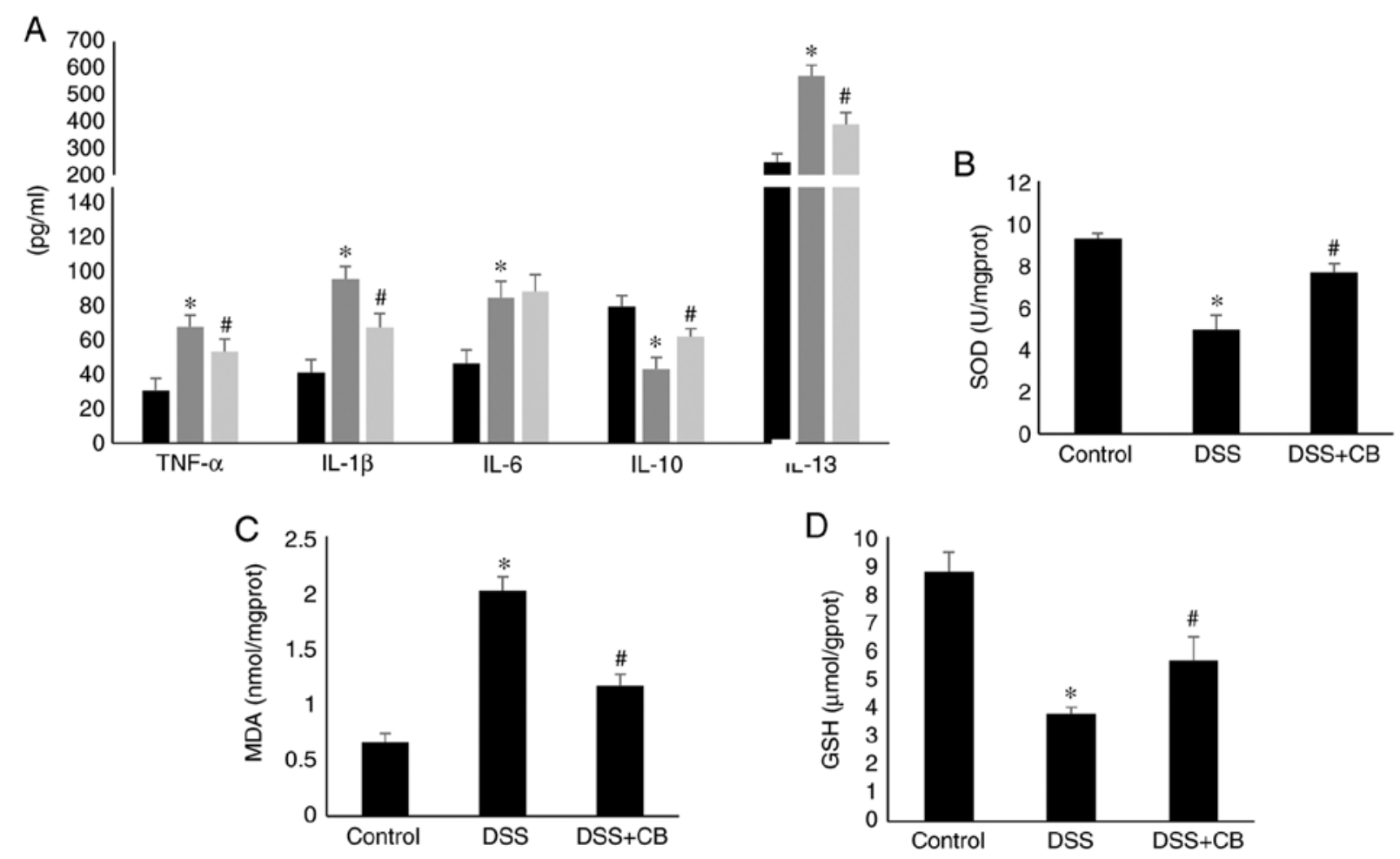

Figure 3. Effect of $C B$ on cytokines secretion levels and oxidative stress indicators. (A) Serum levels of TNF- $\alpha$, IL-1 1 , IL-6, IL-10 and IL-13 in different groups. Levels of oxidative stress indicators, (B) SOD, (C) MDA and (D) GSH in different groups. Data are presented as the mean \pm SD. ${ }^{*} \mathrm{P}<0.05$ vs. control group; "P<0.05 vs. DSS group. CB, Clostridium butyricum; TNF- $\alpha$, tumor necrosis factor $\alpha$; IL, interleukin; DSS, dextran sodium sulfate; MDA, malondialdehyde; GSH, glutathione; SOD, superoxide dismutase.
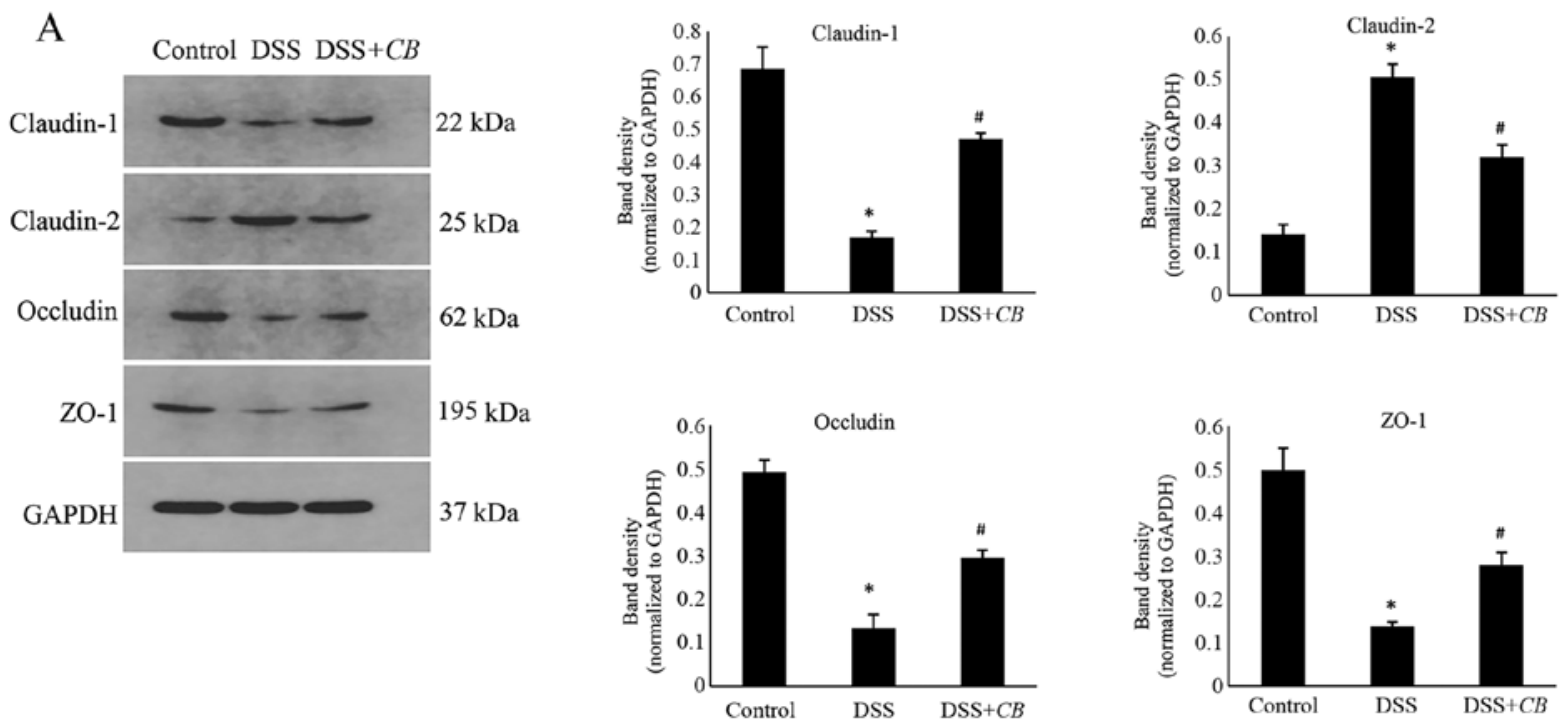

B
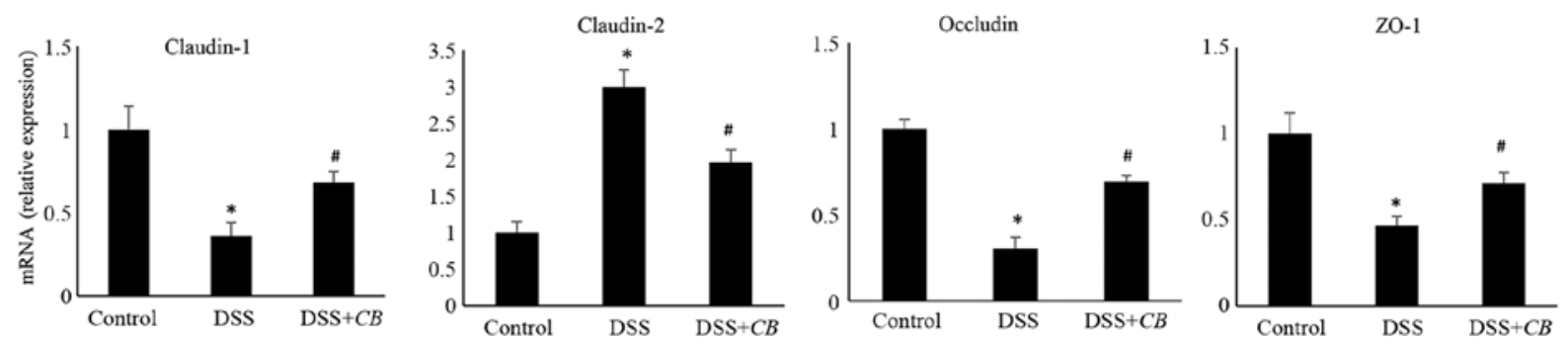

Figure 4. Effects of $C B$ on the expression levels of claudin-1, claudin-2, occludin and ZO-1. (A) Protein expression levels of claudin-1, claudin-2, occludin and ZO-1 in each group were measured using western blot analysis. (B) mRNA expression levels of claudin-1, claudin-2, occludin and ZO-1 were determined using reverse transcription-quantitative PCR. Data are presented as the mean $\pm \mathrm{SD}$. ${ }^{*} \mathrm{P}<0.05$ vs. control group; ${ }^{\text {}} \mathrm{P}<0.05$ vs. DSS group. ZO-1, zona occludens protein 1; $C B$, Clostridium butyricum; DSS, dextran sodium sulfate. 
A

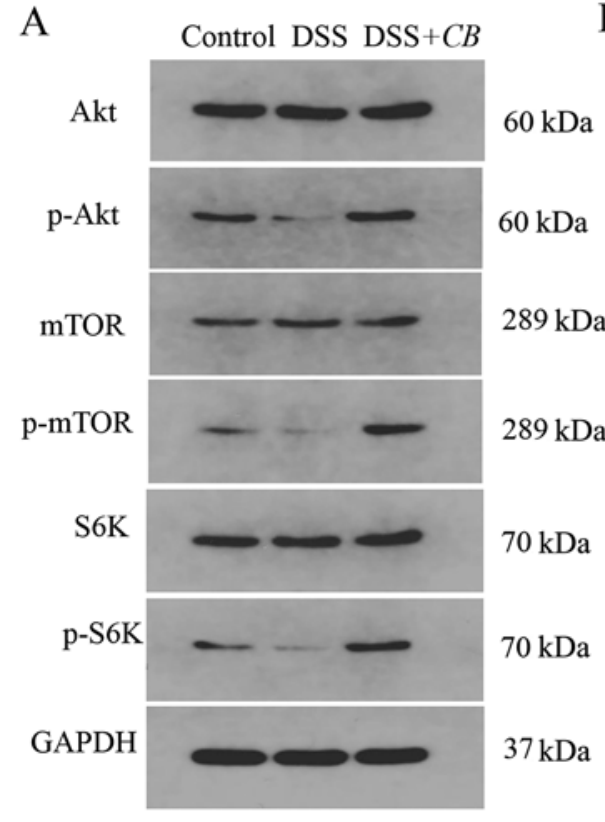

B
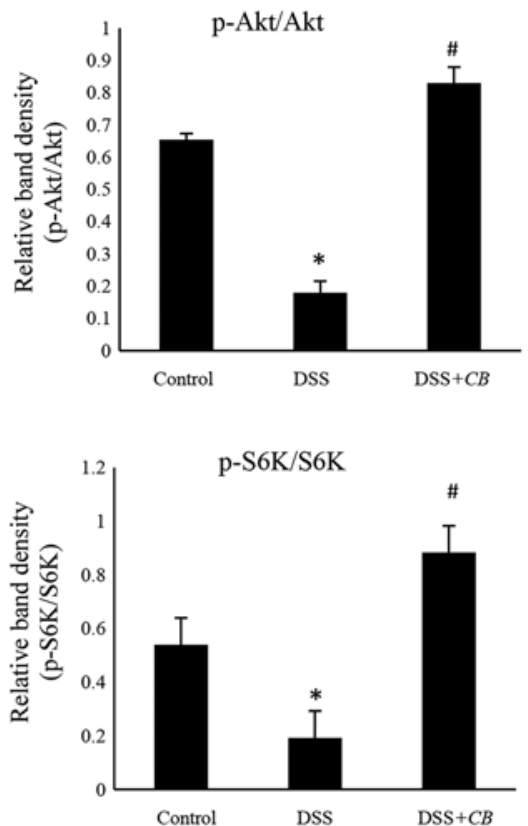
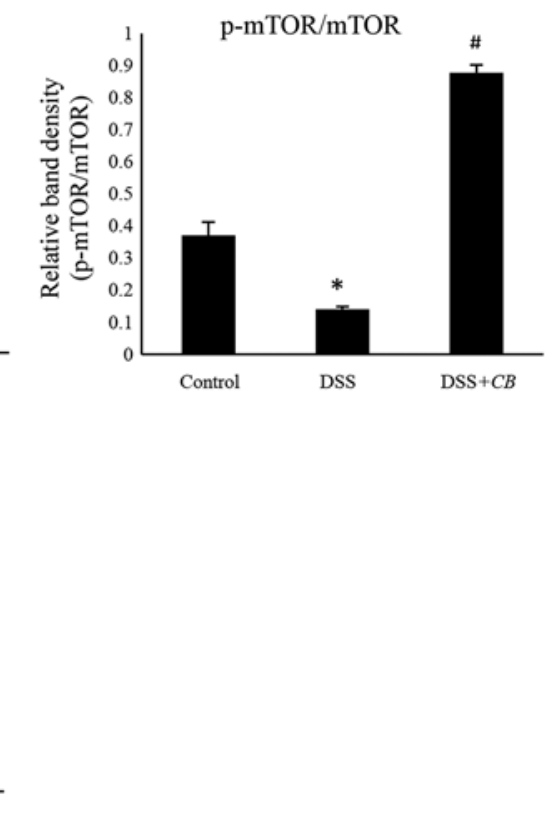

Figure 5. Effect of $C B$ on the Akt/mTOR signaling pathway. (A) Representative western blot analysis results of Akt, mTOR and S6K. (B) Comparison of semi-quantitative results. Data are presented as the mean $\pm \mathrm{SD}$. ${ }^{*} \mathrm{P}<0.05$ vs. control group; ${ }^{*} \mathrm{P}<0.05$ vs. DSS group. CB, Clostridium butyricum; DSS, dextran sodium sulfate; S6K, p70 ribosomal protein S6 kinase; p-, phosphorylated.

microbial balance in the intestine, while exhibiting a high tolerance to several antibiotics $(30,31)$. Therefore, present study aimed to investigate the specific molecular mechanisms of $C B$ in protecting the intestinal mucosal barrier function in DSS-induced colitis.

Currently, the DSS-induced colitis model remains the most widely used mouse model of colitis mimicking human UC due to its similarities in terms of symptoms and histopathological changes (19). In the present study, C57BL/6 mice were treated with $3 \%$ DSS for 7 days to establish the acute colitis mouse model. Colitis in the DSS-induced model was accompanied by several symptoms, including diarrhea, weight loss, hematochezia and histological damage, such as surface epithelial loss, crypt destruction and increased inflammatory cell infiltration. The present results demonstrated that co-treatment of DSS-treated mice with $C B$ relieved colitis symptoms and protected intestinal barrier function. Determining body weight, DAI and histological grading score in mice in the DSS, DSS $+C B$ and control groups identified that $C B$ not only alleviated colitis-related symptoms, maintained body weight and reduced DAI score, but also prevented histological damage by decreasing neutrophil infiltration, promoting the restoration of epithelial cell damage and reducing the rectal histological score. Furthermore, FD-4 serum levels were significantly decreased in the DSS $+C B$ group compared with the DSS group.

Proinflammatory cytokines are associated with the intestinal barrier dysfunction in UC. For example, it has been reported that TNF- $\alpha$ increases TJ permeability by modulating myosin light chain kinase (MLCK) gene expression via the $\mathrm{NF}-\kappa \mathrm{B}$ signaling pathway in Caco-2 cells (32). In addition, IL-1 $\beta$ enhances intestinal TJ permeability by regulating $\mathrm{p} 38$ kinase activation of activating transcription factor 2 (ATF-2) and via ATF-2 regulation of MLCK gene activity (33). IL-13 is considered an important effector cytokine in patients with UC, which may lead to epithelial barrier dysfunction by regulating epithelial apoptosis, TJs and restitution velocity (34). IL-10, an anti-inflammatory cytokine, serves a key role in the intestinal immune system and is closely associated with IBD. A previous study has shown that $\mathrm{IL}_{10}{ }^{-/-}$mice spontaneously develop colitis, which may be alleviated by the administration of IL-10 (35). Furthermore, a genome-wide association study in humans revealed that a single-nucleotide polymorphism in the IL-10 gene was closely associated with IBD (36). Kuhn et al (37), demonstrated in two murine models of bowel injury that IL-6 was induced soon after injury in multiple cell types, while its inhibition resulted in impaired wound healing. Additionally, these authors found that IL-6 mRNA transcripts were enriched within the surrounding area of colon perforation in human patients, thus suggesting that this IL-6-driven wound healing mechanism could be important in humans. Previous studies have reported that butyrate inhibits the secretion of TNF- $\alpha$, interferon- $\gamma$, IL-12, IL-5 and IL-13 $(38,39)$, while $C B$ promotes IL-10 production via intestinal macrophages in the inflamed mucosa of mice with experimental colitis (28). In the present study, treatment with $C B$ also inhibited the expression levels of the proinflammatory cytokines TNF- $\alpha$, IL- $1 \beta$ and IL-13, and promoted that of IL-10. Consistent with previous studies, the current results suggested that IL- 6 secretion levels in mice with DSS-induced colitis were increased compared with the control group. However, the differences in IL-6 secretion levels between the DSS and DSS $+C B$ groups were not significant, indicating that $C B$ had no significant effect on IL-6 secretion. Therefore, the role of IL-6 in wound healing should be further investigated.

MDA, a marker for lipid peroxidation, is commonly used as an indicator for oxidative damage to cells and tissues (40). The present study demonstrated that in DSS-treated animals, 
MDA levels were increased, while co-treatment with $C B$ significantly alleviated its levels. Furthermore, GSH, an important indicator of the body's antioxidant capacity, contributes to the antioxidant defense system (41). It has also been reported that GSH eliminates free radicals and protects the gastrointestinal mucosal epithelium by preventing injuries caused by inflammation, local ischemia and oxidants (42). SOD is an important antioxidant enzyme that attenuates tissue oxidative damage and acts as a free radical scavenger in defense against oxidative cell injury (43). In the current study it was identified that co-treatment of DSS-treated mice with $C B$ significantly elevated GSH levels and SOD enzyme activities.

Inflammation and oxidative stress are two closely associated and tightly linked processes that provoke cell injury (44). It has been reported that the anti-inflammatory and antioxidative effects of $C B$ may be associated with the metabolism of SCFAs. These molecules serve a critical role in colonic health by acting as important energetic substrates for epithelial cells (45), modulating immune cell adhesion molecules and affecting chemotaxis, cytokine release and leukocyte migration (46). In addition, SCFAs may modulate oxidative stress via inhibiting histone deacetylases (47). An in vitro study has shown that butyrate protects colonocytes from reactive oxygen species-induced DNA damage (48). The present study demonstrated that $C B$, as a major source of butyrate, inhibited the secretion of proinflammatory cytokines and reduced oxidative stress.

The present results suggested that $C B$ could protect the intestinal barrier function via upregulating the expression of TJ-related proteins, including claudin-1, occluding and $\mathrm{ZO}-1$, at both protein and mRNA levels. However, the mRNA and protein levels of claudin-2 were decreased in the DSS $+C B$ group. Claudins serve an important role in intestinal barrier function and regulate the permeability of epithelial layers to ions and water by upregulating the expression of $\geq 1$ of the 27 claudin isoforms (49). Each claudin molecule consists of four right helical folded transmembrane domains and two extracellular rings (50). The amino- and carboxyl-terminal groups reside in the cytosol and are linked to ZOs via the post-synaptic density 95/Drosophila discs large/zona-occludens 1 binding domain. Moreover, these two extracellular rings are the structural basis of TJs and affect the selective permeability to ions and water (50). Previous studies have reported that claudin-1, $-3,-4,-5$ and -8 exhibit sealing functions and protect the intestinal barrier function (51-55). Additionally, claudin-2, $-7,-12$ and -15 provide paracellular channels to promote the charge-selective passage of small ions and increase the permeability of intestinal mucosa (56-58).

Occludin regulates the localization and function of TJ complex via phosphorylation, which affects TJ permeability. Furthermore, two subtypes of occludin have been identified, characterized by the formation of two outer rings via four transmembrane domains; these extracellular rings are responsible for binding between adjacent cells (59). Amino acid residues in the intracellular domain of occludin directly bind to ZOs and subsequently interact with cytoskeleton proteins via cytoplasmic proteins (59). ZOs belong to the membrane associated guanylate kinase family and consists of three isoforms: ZO-1,
-2 and -3 . ZOs interact with several transmembrane proteins, and actin cytoskeleton and cytoskeleton-associated proteins via the $\mathrm{N}$ - and $\mathrm{C}$-terminal regions, respectively (1). A previous study has revealed that $C B$ upregulated ZO-1, claudin-1 and occludin expression levels in vitro in lipopolysaccharide (LPS)-treated IPEC-J2 cells and inhibited LPS-induced apoptosis (60). Moreover, Daly and Shirazi-Beeche (61) observed that butyrate downregulated claudin-2 expression in butyrate-treated colonic epithelial cells, using microarray analysis. Consistent with previous studies, the present results suggested that the protein and mRNA expression levels of claudin-1, occludin and ZO-1 were significantly increased in the $C B$-treated group. Furthermore, claudin-2 expression was higher in the DSS group compared with the control group, while $C B$ could reverse this trend.

Akt, a serine/threonine kinase, is an essential intracellular signaling molecule that promotes cell survival, growth, proliferation and metabolism. It has also been reported that activation of Akt may lead to phosphorylation of mTOR, which in turn activates the downstream signaling molecules, S6K and 4E-BP1 (62). Furthermore, activated S6K and 4E-BP1 directly regulate protein synthesis (63). Previous studies have revealed that the increased expression of TJ-related proteins in intestinal epithelial cells is closely associated with the activation of the Akt/mTOR signaling pathway (64). Yan et al (65) investigated the effect of butyrate on TJ permeability in a model of LPS-induced inflammation in IPEC-J2 cells, and found that butyrate elevated both mRNA and protein expression levels of TJ-related proteins via activating the Akt/mTOR-mediated protein synthesis. In addition, a recent study showed that microbiota metabolites, SCFA, activated the mTOR signaling pathway and promoted intestinal epithelial cell expression of RegIIIr and $\beta$-defensins in a $\mathrm{G}$ protein-coupled receptor 43 -dependent manner (66). In the present study, p-Akt and total Akt protein expression levels were alleviated in DSS-treated mice, thus resulting in p-mTOR and $\mathrm{p}$-S6K downregulation. This finding indicated that the Akt/mTOR signaling pathway was inhibited in DSS-induced colitis, and as a result, TJ-related protein synthesis was inhibited, leading to the disruption of the epithelial barrier function. However, treatment with $C B$ restored p-Akt, p-mTOR and p-S6K protein expression levels, suggesting that $C B$ could promote TJ-related protein synthesis via activating the $A k t / m-T O R$ signaling pathway.

The current study has certain limitations. It remains to be investigated whether oral administration of $C B$ increased the content of intestinal SCFAs or affected the gut microbiome. The safety of $C B$ also requires further study. Moreover, animal studies have indicated that $C B$ and SCFAs have a beneficial impact on colonic diseases; however, evidence in humans is currently lacking. Therefore, future studies are required to further determine the effects of combination therapy with anti-inflammatory drugs, prebiotics or probiotics in clinical trials. Due to funding constraints, the protective effect of $C B$ on intestinal functional barrier will require further research.

In conclusion, previous studies have shown that butyrate exhibits anti-inflammatory, barrier-protective and anti-carcinogenic effects in colon. The present results further demonstrated that $C B$ alleviated colon inflammation 
and restored intestinal barrier function in DSS-induced colitis, by promoting TJ-related proteins expression via the activation of the Akt/mTOR-mediated protein synthesis pathway.

\section{Acknowledgements}

Not applicable.

\section{Funding}

This study was supported by the National Natural Science Foundation of China (grant no. 81800481).

\section{Availability of data and materials}

The datasets used and/or analyzed during the current study are available from the corresponding author on reasonable request.

\section{Authors' contributions}

TD designed the experiments, interpreted and analyzed the data, and revised the manuscript. ML and WX designed and performed the experiments, and wrote the manuscript. XW was responsible for data and statistical analyses. All authors read and approved the final manuscript.

\section{Ethics approval and consent to participate}

All experiments in the present study were performed in accordance with the Guidelines on Animal Experiments from The Committee of Medical Ethics, The National Health Department of China. This study and all experimental protocols were approved by the Laboratory Animals Welfare and Ethics Committee of Renmin Hospital of the Wuhan University (approval no. 20190608).

\section{Patient consent for publication}

Not applicable.

\section{Competing interests}

The authors declare that they have no competing interests.

\section{References}

1. Suzuki T: Regulation of intestinal epithelial permeability by tight junctions. Cell Mol Life Sci 70: 631-659, 2013.

2. Sanchez de Medina F, Romero-Calvo I, Mascaraque C and Martinez-Augustin O: Intestinal inflammation and mucosal barrier function. Inflamm Bowel Dis 20: 2394-2404, 2014.

3. Suzuki T: Regulation of the intestinal barrier by nutrients: The role of tight junctions. Anim Sci J 91: e13357, 2020.

4. Andrews C, McLean MH and Durum SK: Cytokine tuning of intestinal epithelial function. Front Immunol 9: 1270, 2018.

5. Rubin DT, Ananthakrishnan AN, Siegel CA, Sauer BG and Long MD: ACG Clinical Guideline: Ulcerative colitis in adults. Am J Gastroenterol 114: 384-413, 2019.

6. Kornbluth A and Sachar DB: Ulcerative colitis practice guidelines in adults: American College Of Gastroenterology, Practice Parameters Committee. Am J Gastroenterol 105: 501-523; quiz 524,2010 .
7. Chang J, Leong RW, Wasinger VC, Ip M, Yang M and Phan TG: Impaired intestinal permeability contributes to ongoing bowel symptoms in patients with inflammatory bowel disease and mucosal healing. Gastroenterology 153: 723-731.e1, 2017.

8. Luissint AC, Parkos CA and Nusrat A: Inflammation and the intestinal barrier: Leukocyte-epithelial cell interactions, cell junction remodeling, and mucosal repair. Gastroenterology 151: 616-632, 2016

9. Buckley A and Turner JR: Cell biology of tight junction barrier regulation and mucosal disease. Cold Spring Harb Perspect Biol 10: a029314, 2018.

10. Khor B, Gardet A and Xavier RJ: Genetics and pathogenesis of inflammatory bowel disease. Nature 474: 307-317, 2011.

11. Suau A, Bonnet R, Sutren M, Godon JJ, Gibson GR, Collins MD and Doré J: Direct analysis of genes encoding 16S rRNA from complex communities reveals many novel molecular species within the human gut. Appl Environ Microbiol 65: 4799-4807, 1999.

12. Nicholson JK, Holmes E, Kinross J, Burcelin R, Gibson G, Jia W and Pettersson S: Host-gut microbiota metabolic interactions. Science 336: 1262-1267, 2012.

13. Hill C, Guarner F, Reid G, Gibson GR, Merenstein DJ, Pot B, Morelli L, Canani RB, Flint HJ, Salminen S, et al: Expert consensus document. The international scientific association for probiotics and prebiotics consensus statement on the scope and appropriate use of the term probiotic. Nat Rev Gastroenterol Hepatol 11: 506-514, 2014.

14. Schönherr-Hellec S, Klein G, Delannoy J, Ferraris L, Friedel I, Rozé JC, Butel MJ and Aires J: Comparative phenotypic analysis of 'Clostridium neonatale' and Clostridium butyricum isolates from neonates. Anaerobe 48: 76-82, 2017.

15. Kong Q, He GQ, Jia JL, Zhu QL and Ruan H: Oral administration of Clostridium butyricum for modulating gastrointestinal microflora in mice. Curr Microbiol 62: 512-517, 2011.

16. Wang HB, Wang PY, Wang X, Wan YL and Liu YC: Butyrate enhances intestinal epithelial barrier function via up-regulation of tight junction protein Claudin-1 transcription. Dig Dis Sci 57: 3126-3135, 2012

17. Peng L, Li ZR, Green RS, Holzman IR and Lin J: Butyrate enhances the intestinal barrier by facilitating tight junction assembly via activation of AMP-activated protein kinase in Caco-2 cell monolayers. J Nutr 139: 1619-1625, 2009.

18. Manning BD and Cantley LC: AKT/PKB signaling: Navigating downstream. Cell 129: 1261-1274, 2007.

19. Wirtz S, Popp V, Kindermann M, Gerlach K, Weigmann B, Fichtner-Feigl S and Neurath MF: Chemically induced mouse models of acute and chronic intestinal inflammation. Nat Protoc 12: 1295-1309, 2017.

20. Wang Y, Gu Y, Fang K, Mao K, Dou J, Fan H, Zhou C and Wang H: Lactobacillus acidophilus and Clostridium butyricum ameliorate colitis in murine by strengthening the gut barrier function and decreasing inflammatory factors. Benef Microbes 9: 775-787, 2018.

21. Dawson PA, Huxley S, Gardiner B, Tran T, McAuley JL, Grimmond S, McGuckin M and Markovich D: Reduced mucin sulfonation and impaired intestinal barrier function in the hyposulfataemic NaS1 null mouse. Gut 58: 910-919, 2009.

22. Dieleman LA, Palmen MJ, Akol H, Bloemena E, Peña AS, Meuwissen SG and Van Rees EP: Chronic experimental colitis induced by dextran sulphate sodium (DSS) is characterized by Th1 and Th2 cytokines. Clin Exp Immunol 114: 385-391, 1998.

23. Livak KJ and Schmittgen TD: Analysis of relative gene expression data using real-time quantitative PCR and the 2(-Delta Delta C(T)) method. Methods 25: 402-408, 2001

24. Matsuoka $\mathrm{K}$ and Kanai $\mathrm{T}$ : The gut microbiota and inflammatory bowel disease. Semin Immunopathol 37: 47-55, 2015.

25. Zeissig S, Bürgel N, Günzel D, Richter J, Mankertz J, Wahnschaffe U, Kroesen AJ,Zeitz M, Fromm M and Schulzke JD: Changes in expression and distribution of claudin 2,5 and 8 lead to discontinuous tight junctions and barrier dysfunction in active Crohn's disease. Gut 56: 61-72, 2007.

26. Martini E, Krug SM, Siegmund B, Neurath MF and Becker C: Mend your fences: The epithelial barrier and its relationship with mucosal immunity in inflammatory bowel disease. Cell Mol Gastroenterol Hepatol 4: 33-46, 2017.

27. Okamoto T, Sasaki M, Tsujikawa T, Fujiyama Y, Bamba T and Kusunoki M: Preventive efficacy of butyrate enemas and oral administration of Clostridium butyricum M588 in dextran sodium sulfate-induced colitis in rats. J Gastroenterol 35: $341-346,2000$ 
28. Hayashi A, Sato T, Kamada N, Mikami Y, Matsuoka K, Hisamatsu T, Hibi T, Roers A, Yagita H, Ohteki T, et al: A single strain of Clostridium butyricum induces intestinal IL-10-producing macrophages to suppress acute experimental colitis in mice. Cell Host Microbe 13: 711-722, 2013.

29. Kanai T, Mikami Y and Hayashi A: A breakthrough in probiotics: Clostridium butyricum regulates gut homeostasis and anti-inflammatory response in inflammatory bowel disease. J Gastroenterol 50: 928-939, 2015

30. Seki H, Shiohara M, Matsumura T, Miyagawa N, Tanaka M, Komiyama A and Kurata S: Prevention of antibiotic-associated diarrhea in children by Clostridium butyricum MIYAIRI. Pediatr Int 45: 86-90, 2003.

31. Chen L, Li S, Zheng J, Li W, Jiang X, Zhao X, Li J, Che L, Lin Y, $\mathrm{Xu} \mathrm{S}$, et al: Effects of dietary Clostridium butyricum supplementation on growth performance, intestinal development, and immune response of weaned piglets challenged with lipopolysaccharide. J Anim Sci Biotechnol 9: 62, 2018.

32. Ye D and Ma TY: Cellular and molecular mechanisms that mediate basal and tumour necrosis factor-alpha-induced regulation of myosin light chain kinase gene activity. J Cell Mol Med 12: 1331-1346, 2008 .

33. Al-Sadi R, Guo S, Ye D, Dokladny K, Alhmoud T, Ereifej L, Said HM and Ma TY: Mechanism of IL-1 $\beta$ modulation of intestinal epithelial barrier involves p38 kinase and activating transcription factor-2 activation. J Immunol 190: 6596-6606, 2013.

34. Heller F, Florian P, Bojarski C, Richter J, Christ M, Hillenbrand B, Mankertz J, Gitter AH, Bürgel N, Fromm M, et al: Interleukin-13 is the key effector Th2 cytokine in ulcerative colitis that affects epithelial tight junctions, apoptosis, and cell restitution. Gastroenterology 129: 550-564, 2005.

35. Kühn R, Löhler J, Rennick D, Rajewsky K and Müller W: Interleukin-10-deficient mice develop chronic enterocolitis Cell 75: 263-274, 1993.

36. Franke A, Balschun T, Karlsen TH, Sventoraityte J, Nikolaus S, Mayr G, Domingues FS, Albrecht M, Nothnagel M Ellinghaus D, et al: Sequence variants in IL10, ARPC2 and multiple other loci contribute to ulcerative colitis susceptibility. Nat Genet 40: 1319-1323, 2008.

37. Kuhn KA, Manieri NA, Liu TC and Stappenbeck TS: IL-6 stimulates intestinal epithelial proliferation and repair after injury. PLoS One 9: e114195, 2014

38. Inan MS, Rasoulpour RJ, Yin L, Hubbard AK, Rosenberg DW and Giardina C: The luminal short-chain fatty acid butyrate modulates NF-kappaB activity in a human colonic epithelial cell line. Gastroenterology 118: 724-734, 2000.

39. Nancey S, Bienvenu J, Coffin B, Andre F, Descos L and Flourie B Butyrate strongly inhibits in vitro stimulated release of cytokines in blood. Dig Dis Sci 47: 921-928, 2002.

40. Mano T, Sinohara R, Sawai Y, Oda N, Nishida Y, Mokumo T, Asano K, Ito Y, Kotake M, Hamada M, et al: Changes in lipid peroxidation and free radical scavengers in the brain of hyperand hypothyroid aged rats. J Endocrinol 147: 361-365, 1995.

41. Orient A, Donkó A, Szabó A, Leto TL and Geiszt M: Novel sources of reactive oxygen species in the human body. Nephrol Dial Transplant 22: 1281-1288, 2007.

42. Mårtensson J, Jain A and Meister A: Glutathione is required for intestinal function. Proc Natl Acad Sci USA 87: 1715-1719, 1990.

43. Kyle ME, Miccadei S, Nakae D and Farber JL: Superoxide dismutase and catalase protect cultured hepatocytes from the cytotoxicity of acetaminophen. Biochem Biophys Res Commun 149: 889-896, 1987

44. Tian T, Wang $Z$ and Zhang J: Pathomechanisms of oxidative stress in inflammatory bowel disease and potential antioxidant therapies. Oxid Med Cell Longev 2017: 4535194, 2017.

45. Kelly CJ, Zheng L, Campbell EL, Saeedsolz CC, Bayless AJ, Wilson KE, Glover LE, Kominsky DJ, Magnuson A, et al: Crosstalk between microbiota-derived short-chain fatty acids and intestinal epithelial HIF augments tissue barrier function. Cell Host Microbe 17: 662-671, 2015.

46. Kaczmarczyk MM, Miller MJ and Freund GG: The health benefits of dietary fiber: Beyond the usual suspects of type 2 diabetes mellitus, cardiovascular disease and colon cancer. Metabolism 61: 1058-1066, 2012.

47. Shimazu T, Hirschey MD, Newman J, He W, Shirakawa K, Le Moan N, Grueter CA, Lim H, Saunders LR, Stevens RD, et al: Suppression of oxidative stress by beta-hydroxybutyrate, an endogenous histone deacetylase inhibitor. Science 339: 211-214, 2013.
48. Rosignoli P, Fabiani R, De Bartolomeo A, Spinozzi F, Agea E, Pelli MA and Morozzi G: Protective activity of butyrate on hydrogen peroxide-induced DNA damage in isolated human colonocytes and HT29 tumour cells. Carcinogenesis 22: 1675-1680, 2001

49. Citi S: Intestinal barriers protect against disease. Science 359: 1097-1098, 2018.

50. Suzuki H, Tani K and Fujiyoshi Y: Crystal structures of claudins: Insights into their intermolecular interactions. Ann N Y Acad Sci 1397: 25-34, 2017.

51. Inai T, Kobayashi J and Shibata Y: Claudin-1 contributes to the epithelial barrier function in MDCK cells. Eur J Cell Biol 78: 849-855, 1999.

52. Milatz S, Krug SM, Rosenthal R, Günzel D, Müller D, Schulzke JD, Amasheh S and Fromm M: Claudin-3 acts as a sealing component of the tight junction for ions of either charge and uncharged solutes. Biochim Biophys Acta 1798: 2048-2057, 2010.

53. Van Itallie C, Rahner $\mathrm{C}$ and Anderson JM: Regulated expression of claudin-4 decreases paracellular conductance through a selective decrease in sodium permeability. J Clin Invest 107: 1319-1327, 2001

54. Amasheh S, Schmidt T, Mahn M, Florian P, Mankertz J, Tavalali S, Gitter AH, Schulzke JD and Fromm M: Contribution of claudin-5 to barrier properties in tight junctions of epithelial cells. Cell Tissue Res 321: 89-96, 2005.

55. Yu AS, Enck AH, Lencer WI and Schneeberger EE: Claudin- 8 expression in Madin-Darby canine kidney cells augments the paracellular barrier to cation permeation. J Biol Chem 278 17350-17359, 2003

56. Amasheh S, Meiri N, Gitter AH, Schöneberg T, Mankertz J, Schulzke JD and Fromm M: Claudin-2 expression induces cation-selective channels in tight junctions of epithelial cells. J Cell Sci 115: 4969-4976, 2002.

57. Alexandre MD, Jeansonne BG, Renegar RH, Tatum R and Chen YH: The first extracellular domain of claudin-7 affects paracellular Cl-permeability. Biochem Biophys Res Commun 357: 87-91, 2007.

58. Fujita H, Chiba H, Yokozaki H, Sakai N, Sugimoto K, Wada T, Kojima T, Yamashita T and Sawada N: Differential expression and subcellular localization of claudin-7, $-8,-12,-13$, and -15 along the mouse intestine. J Histochem Cytochem 54: 933-944, 2006

59. Dörfel MJ and Huber O: Modulation of tight junction structure and function by kinases and phosphatases targeting occludin. J Biomed Biotechnol 2012: 807356, 2012.

60. Xiao Z, Liu L, Tao W, Pei X, Wang G and Wang M: Clostridium tyrobutyricum protect intestinal barrier function from LPS-induced apoptosis via P38/JNK signaling pathway in IPEC-J2 Cells. Cell Physiol Biochem 46: 1779-1792, 2018.

61. Daly K and Shirazi-Beechey SP: Microarray analysis of butyrate regulated genes in colonic epithelial cells. DNA Cell Biol 25: 49-62, 2006.

62. Kandel ES and Hay N: The regulation and activities of the multifunctional serine/threonine kinase Akt/PKB. Exp Cell Res 253 210-229, 1999.

63. Manning BD and Toker A: AKT/PKB signaling: Navigating the network. Cell 169: 381-405, 2017.

64. Wang H, Ji Y, Wu G, Sun K, Sun Y, Li W, Wang B, He B, Zhang Q, Dai $Z$ and Wu Z: 1-tryptophan activates mammalian target of rapamycin and enhances expression of tight junction proteins in intestinal porcine epithelial cells. J Nutr 145: 1156-1162, 2015.

65. Yan $\mathrm{H}$ and Ajuwon KM: Butyrate modifies intestinal barrie function in IPEC-J2 cells through a selective upregulation of tight junction proteins and activation of the Akt signaling pathway. PLoS One 12: e0179586, 2017.

66. Zhao Y, Chen F, Wu W, Sun M, Bilotta AJ, Yao S, Xiao Y, Huang X, Eaves-Pyles TD, Golovko G, et al: GPR43 mediates microbiota metabolite SCFA regulation of antimicrobial peptide expression in intestinal epithelial cells via activation of mTOR and STAT3. Mucosal Immunol 11: 752-762, 2018.

This work is licensed under a Creative Commons Attribution-NonCommercial-NoDerivatives 4.0 International (CC BY-NC-ND 4.0) License. 